\title{
Delimitación del Constructo Voluntad de Trabajo
}

\section{Demarcation of the Will-To-Work Construct}

\author{
Gabriela Susana Lozzia*, Facundo Juan Pablo Abal, María Ester Aguerri, \\ María Silvia Galibert y Horacio Félix Atoréis \\ Universidad de Buenos Aires, Argentina
}

(Recepción: Octubre 2007 - Aceptación: Noviembre 2007)

\begin{abstract}
Resumen
Ante la queja habitual de los docentes universitarios acerca de la poca perseverancia y disposición al esfuerzo de sus alumnos, se buscó definir conceptualmente el rasgo Voluntad de Trabajo (VT) para la construcción de una escala. Se vinculó con el Modelo de Control de la Acción de Kuhl. Se estudiaron los Modelos de Personalidad (Guilford, Cattell, Eysenck, Cinco Grandes y Zuckerman) para identificar las dimensiones relacionadas y sus indicadores. La VT se caracterizó como la actitud por la cual un individuo se aboca a sus obligaciones con responsabilidad, eficiencia, automotivación y sin dilaciones. Se relacionó con la capacidad para generar procesos volitivos que lo comprometan en el inicio, continuación y concreción de una tarea sin que tendencias contrarias logren desviarlo.
\end{abstract}

Palabras claves: voluntad de trabajo - perseverancia - estudiantes universitarios

\begin{abstract}
Due to the usual complaint from professors about the little perseverance and disposition to effort in their university students, a conceptual definition of the Will- to-Work feature (WW) was sought so that a scale could be constructed. WW was defined according to Kuhl's Action Control Model. Personality Models (Guilford, Cattell, Eysenck, Big Five and Zuckerman) were studied to identify the related dimensions and their indicators. WW was defined as the attitude by which an individual becomes devoted to his/ her obligations with responsibility, efficiency, self-motivation and promptness. This was explained by the capacity to generate volitive processes that commit him/her at the beginning, development and materialization of a task without opposing tendencies that might divert his/her focus from the task in question.
\end{abstract}

Key words: will-to-work - perseverance - university students

\footnotetext{
${ }^{1}$ La investigación que se presenta en este artículo fue realizada con subsidios de la Universidad de Buenos Aires (UBACyT P020) y de la Agencia Nacional de Promoción Científica y Tecnológica (ANPCyT PICT 2004 Nº 20909)

* Correspondencia a: Gabriela Susana Lozzia. Instituto de Investigaciones de la Facultad de Psicología de la Universidad de Buenos Aires. Dirección: Av. Lope de Vega 1507 Dpto. 2 (1407), Buenos Aires, Argentina. Teléfono: (011)4568-6172 E-mail: glozzia@psi.uba.ar
} 


\section{Delimitación del Constructo Voluntad de Trabajo}

En estos tiempos de debate educativo es común escuchar que la educación viene en caída libre. Muchos de los problemas que antes parecían exclusividad de la escuela media están apareciendo en el nivel universitario. Si bien no se ha perdido el respeto por la autoridad, ni se puede decir que los alumnos universitarios carezcan de límites, se ha vuelto cada vez más frecuente observar una menor disposición al esfuerzo y una inclinación al facilismo en el alumnado. El empeño que implica la formación de grado aparece relegado a un segundo plano, cuando no está ausente. Cada vez son más los profesores a los que les llama la atención el bajo nivel de lectura del material obligatorio, la preferencia por leer y estudiar resúmenes realizados por otras personas, la poca motivación de los alumnos para la realización de las actividades, la escasa participación en clase, la tendencia a postergar las obligaciones académicas y la falta de perseverancia, reconocible cuando abandonan la carrera cursada por no haber obtenido la calificación deseada.

Son múltiples las causas que han podido generar estos cambios en las conductas de los estudiantes y responden a diversos planos: sociales, culturales y psicológicos. Pero lo cierto es que las repercusiones que han tenido estos fenómenos han propiciado diferencias individuales que hacen que algunos alumnos actúen de forma responsable y perseverante mientras que otros no.

Esta problemática ha despertado el interés de los autores de este artículo en conseguir un instrumento que sirviera para objetivar, en la medida de lo posible, este aspecto de la personalidad. Pero la mayoría de los constructos desarrollados para definir este rasgo son operacionalizados con extensos cuestionarios que proporcionan una medida global de la personalidad y cuyas propiedades psicométricas se verían modificadas al intentar la administración exclusiva de los ítems de interés. En cambio otros cuestionarios de menor alcance como Motivación hacia el Logro de McClelland (1961) y el MAPE-1 de Alonso-Tapia (1992) no se ajustan estrictamente a la variable que se pretende evaluar; el primero por incluir indicadores que reflejan una búsqueda activa de desafíos y de reconocimiento que exceden el dominio de lo requerido y el segundo por estar adaptado para una población infantil.

La ausencia de una delimitación teórica y una operacionalización compatibles con lo que se desea estudiar impuso la necesidad de definir el constructo y elaborar una prueba ad-hoc para futuras investigaciones. Se puso como condición que la misma sea unidimensional y corta para reducir el tiempo de administración.

El objetivo de este trabajo es alcanzar una definición conceptual para un rasgo al que se denominará Voluntad de Trabajo (VT), necesaria para construir una escala adaptada a una población de alumnos universitarios. A continuación se presentarán las líneas teóricas que sustentan esta denominación y se delimitará su contribución en un modelo explicativo de la motivación humana. Por último, para precisar las características distintivas de este constructo serán analizadas las definiciones más relevantes recabadas en la literatura de diversos modelos de personalidad.

\section{¿Por qué Voluntad?}

\section{La mirada filosófica.}

La búsqueda de una definición precisa de la Voluntad fue una temática medular en discusiones filosóficas y religiosas durante miles de años. Abbagnano (1974) rescató la perspectiva filosófica que ha identificado la Voluntad con el origen de la acción en general. Por un lado, Descartes pensaba que todos los actos de los hombres no son más que Voluntad; en tanto que Locke la concibió como la potencia para iniciar, continuar o terminar varias acciones en nuestra mente y varios movimientos de nuestro cuerpo sólo con la intervención de un pensamiento. Primero San Agustín y más tarde Duns Escoto la consideraron como el motor que impulsa y dirige el movimiento de todo el reino de las facultades.

Pero también existían otras concepciones filosóficas que pusieron el acento en el carácter racional de la Voluntad. Su relación con el entendimiento ya había sido planteada por los filósofos de la antigüedad (Ferrater Mora, 1975). Platón postuló que, así como el deseo pertenece al orden de 
la sensibilidad, la Voluntad responde al orden del intelecto. Para Aristóteles Voluntad y deseo eran los motores de la conducta, salvo que la primera era de índole racional. Desde esta concepción, la Voluntad se erigía como principio racional de la acción en tanto se la suponía como un apetito conforme al intelecto. Esta división permitía diferenciar al hombre del animal irracional, cuyo móvil comportamental no podía ser ubicado en la Voluntad sino que era localizado en los instintos.

\section{La visión experimental.}

La aplicación de métodos experimentales al estudio de la Voluntad no llegó hasta principios del siglo XX y fue iniciado por la Escuela de Wurzburgo. La existencia de la Voluntad se confirmaba utilizando como método la introspección sistemática. Ya en las primeras y más importantes experiencias llevadas a cabo, los psicólogos modernos buscaron diferenciarse de la tradición filosófica que concebía la Voluntad como una de las facultades del alma. Por tal motivo, se prefirió desestimar esta denominación y sustituirla por querer. Así, las experiencias registraban objetivamente la Voluntad a partir de la realización efectiva de aquello que se quería hacer (Dorsch, 1991). Es decir, como acto de querer, como volición. En el marco de estos estudios, Michotte y Prüm (1910) denominaron acto voluntario a la toma de conciencia de la acción. A su vez, Ach (1910) caracterizó el acto de voluntad interior definido como el proceso psíquico que conducía a la concreción de lo planificado, distinguiéndolo del acto de voluntad exterior que conllevaba el conjunto de movimientos que hacen posible la conducta necesaria.

Estos orígenes inauguraron un camino que sirvió para delinear conceptualmente el comportamiento voluntario como una matriz relacional de funciones cognoscitivas y motivacionales. Sin embargo, la paulatina consolidación de la motivación como constructo básico explicativo de las causas del comportamiento y la creciente hegemonía del conductismo como paradigma experimental ensombrecieron los aportes de estas investigaciones de la Voluntad (Arana \& Sanfeliu, 1994).

\section{El resurgimiento de la Voluntad.}

Durante la década de 1980, diferentes modelos de la motivación (Heckhausen, 1987; Kuhl, 1985) retomaron los aportes de la Escuela de Wurzburgo reubicando en un marco teórico más amplio la función específica que tenía la Voluntad. Este resurgimiento también reflotó el problema de la denominación del constructo. Arana y Mateos (1997) argumentaron que Voluntad podía parecer un concepto corriente y con cierta vaguedad, motivo por el cual, adhiriendo a la línea teórica de la escuela alemana, decidieron nombrarlo como volición o proceso volitivo para evitar malentendidos.

Agrupados bajo el nombre de Teorías de la Acción (Barberá, 2000), estos modelos partieron de la distinción entre la intencionalidad de la conducta y la mediación de los procesos volitivos para hacerla operativa. La intencionalidad supone las tendencias motivacionales que llevan al sujeto a perseguir la meta que se ha propuesto. Empero la determinación de la meta y el compromiso con ella no permiten un pronóstico certero sobre si será o no alcanzada. En particular, el Modelo de Control de la Acción desarrollado por Kuhl (1985) postula la existencia de tendencias de acción alternativas que interfieren el cumplimiento de la intención. Es decir, hay factores tanto internos como externos que ofrecen desvíos tentadores para sujetos poco tenaces. El modelo se completa con la incorporación de modos de control personal que intentan reducir o anular la incidencia de las tendencias contrarias y, al mismo tiempo, facilitan y mantienen la intención. Desde esta perspectiva, la Voluntad es el proceso psíquico que permite dirigir y proteger los impulsos que componen la intención en pos del objetivo limitando la influencia de tendencias contrarias. La visión actual de la Voluntad la concibe como el conjunto de mecanismos que regulan la conducta para lograr la superación de los obstáculos y el mantenimiento de la intención desde el planteo de la meta hasta su concreción. Al decir de Karoly (1993), los procesos volitivos son, en definitiva, procesos de autorregulación de la conducta. Son esencialmente meta-cogniciones implementadas de forma estratégica como argumentos motivacionales que mantienen el objetivo, o bien sirven para estabilizar los estados emocionales hasta alcanzar un equilibrio que favorezca la ejecución.

Los aportes vertidos desde el Modelo del Control de la Acción son altamente compatibles con la clasificación de la Voluntad que Rojas (2003) ha realizado según la forma con que ésta se presenta: 
1. La Voluntad inicial: pone en marcha al individuo, rompe con la inercia y enfoca la intencionalidad en un único sentido.

2. La Voluntad perseverante: cuida y sostiene la intención a lo largo de su desarrollo evitando que tendencias contrarias la afecten. Contar con este tipo de voluntad permite al individuo tomar más riesgos a la hora de embarcarse en proyectos, dado que en ella intervienen factores como el tesón, el empeño y la firmeza para llevar adelante la meta propuesta.

3. La Voluntad capaz de superar frustraciones: se refiere a la capacidad del individuo para sobreponerse a la adversidad. Posibilita retomar la acción cuando el camino de la intención se encuentra obstaculizado.

4. La Voluntad ejemplar: requiere de paciencia y laboriosidad. Supone la implicación directa con aquello que se realiza y por esto se pretende concluir el trabajo atendiendo a cada uno de los detalles. Se alimenta de la satisfacción que genera al sujeto la tarea bien terminada.

Cabe aclarar que la Voluntad capaz de superar frustraciones ha sido dejada de lado para la especificación del constructo por dos razones: a) el carácter eventual atribuible a los impedimentos; y b) porque, según investigaciones actuales, el obstáculo podría producir tanto un efecto disruptivo que perjudica la acción como la activación de los procesos volitivos que acarrearían un beneficio para la ejecución (Arana \& Mateos, 1997).

\section{¿Por qué Voluntad de Trabajo?}

El lenguaje cotidiano e incluso el filosófico han hecho un uso general del concepto Voluntad. También desde la Psiquiatría se la ha definido de manera amplia y por lo negativo a partir de los trastornos de la voluntad. Como tal, esta denominación podría ser aplicable a conductas tan simples como mover un brazo para alcanzar un vaso o conductas mucho más complejas como la preparación de un examen. Es por esta razón que, mientras algunos autores prefirieron utilizar otro nombre, los autores de este artículo optaron por mantenerlo pero restringiendo su significado al acompañarlo con el concepto de Trabajo.

Precisar una definición de Trabajo no resultó tarea sencilla dada la multiplicidad de componentes que presenta y la enorme cantidad de disciplinas que lo incluyen como objeto de estudio. En un intento por alcanzar una definición teórica, Peiró, Prieto y Roe (1996) analizaron y sistematizaron tres aproximaciones que enfatizan diferentes aspectos. La vertiente proveniente de la Psicología Social concibe el trabajo como un fenómeno o realidad resultado del desarrollo del sujeto en interacción con los otros. Otras aproximaciones se centran en el carácter intrínseco - extrínseco de la recompensa. Desde este enfoque, representado principalmente por las Teorías de la Motivación, el trabajo es un medio para obtener un fin. Ya sea para asegurar un sustento vital o para satisfacer necesidades de variada naturaleza, el trabajo se realiza para obtener algo a cambio. Existen definiciones más básicas que describen el trabajo como la acción física y/o mental empleada para realizar una tarea. A esta perspectiva se asocia la medición de los rasgos de personalidad que serán analizados más adelante.

El factor común de todas estas visiones reside en entender al Trabajo como una actividad humana eminentemente intencional y propositiva. Es esta amplia concepción la que sirvió a los fines de esta delimitación teórica. Se adoptó, entonces, la definición integradora de Trabajo propuesta por Peiró et al. (1996):

Conjunto de actividades humanas, retribuidas o no, de carácter productivo y creativo, que mediante el uso de técnicas, instrumentos, materias o información disponibles, permite obtener, producir o prestar ciertos bienes, productos o servicios. En dicha actividad, la persona aporta energías, habilidades, conocimientos, y otros diversos recursos, y obtiene algún tipo de compensación material, psicológica y/o social. (p. $38)$.

Integrando los aportes hasta aquí desarrollados se ha definido la Voluntad de Trabajo como la capacidad de un sujeto para generar procesos volitivos que le permitan comprometerse activamente 
en el inicio de un trabajo, persistir en su concreción sin que las tendencias contrarias logren desviarlo de su objetivo y concluirlo con precisión y puntualidad.

\section{¿Por qué Voluntad de Trabajo como un Rasgo de Personalidad?}

Ahora bien, la capacidad del sujeto de articular los modos de control de la acción propuestos por Kuhl (1985) responde en parte a determinantes específicos de la situación y en parte a características de personalidad del individuo (Barberá, 1995). Es en este segundo punto donde la VT, concebida como un rasgo, se inserta en el modelo motivacional.

Seguidamente se mostrará cómo distintos modelos de personalidad reconocidos aluden parcialmente a este constructo al que se ha llamado VT. Su análisis contribuirá a especificarla como un rasgo identificable desde diferentes paradigmas y a evaluarla desde los distintos instrumentos que se han desarrollado para medirlo. Al mismo tiempo, aportará nuevos elementos para precisar tanto la definición conceptual como la operacional.

Este estudio comparativo comienza por las tres grandes teorías de la personalidad: la teoría de Guilford, la teoría de Cattell y la teoría de Eysenck. Luego se estudiará el modelo pentafactorial que, según Digman (1990), parece contener las ideas principales de estas tres teorías clásicas. Por último, se integrarán los aportes del modelo pentafactorial alternativo propuesto, más recientemente, por Zuckerman.

\section{El Modelo de Personalidad de Guilford.}

Guilford (1959) partió de la presunción de que un rasgo es una forma distinguible, relativamente perdurable, en la que un individuo se diferencia de otro. Esta amplia definición le permitió considerar a la personalidad como un patrón único de rasgos somáticos, motivacionales, aptitudinales y temperamentales. Estos últimos surgieron tras sus estudios factoriales de la inteligencia. Guilford descubrió que algunos de los factores no eran asimilables a los elementos intelectuales sino que respondían al campo del temperamento. Para la construcción de su cuestionario Guilford-Zimmermann Temperament Survey (GZTS, Guilford \& Zimmerman, 1949) partió, por un lado, de las escalas ya existentes y, por otro, de las descripciones clínicas de determinados tipos de personalidad (por ejemplo, introversión/extraversión). Estos estudios ceñidos a los rasgos temperamentales de la personalidad le permitieron delimitar una estructura jerárquica constituida por rasgos de primer y segundo orden que, si bien se mantuvo como modelo básico, fue incorporando diversos factores a medida que avanzaban sus investigaciones. La última versión del GZTS (Guilford, 1975) se compone de diez escalas: Actividad General (G), Ascendencia (A), Sociabilidad (S), Estabilidad Emocional (E), Objetividad (O), Retraimiento (R), Cordialidad (F), Reflexividad (T), Cooperación (P) y Masculi$\operatorname{nidad}(\mathrm{M})$.

A pesar de que no se encuentra en su modelo un rasgo que refiera directamente a la VT, es posible mencionar como parcialmente relacionados a los rasgos primarios de Actividad General y Retraimiento. Del conjunto de disposiciones que componen el primer rasgo se rescatan los que aluden a la energía con que se realizan las actividades en contraposición a una falta de vitalidad o fatiga. Asimismo son destacables los que se refieren a la constancia que se imprime en las acciones evitando posibles interrupciones en busca de un descanso. El Retraimiento está relacionado con el autocontrol y la seriedad (entendida como contraria a la despreocupación) así como también con la capacidad de realizar un esfuerzo persistente. Sin lugar a duda, la VT integra estos aportes dado que sin energía no hay impulso para la acción y sin autocontrol no existe posibilidad de sostener y encaminar la energía en beneficio propio; pero no los confunde con otros aspectos a los que hacen referencia por un lado, la faceta de Actividad General, como son el entusiasmo y el gusto por la velocidad en contra de un ritmo pausado y, por el otro, la dimensión de Retraimiento, que incluye la inhibición de impulsos en general.

En la actualidad, el modelo de Guilford se halla debilitado por la falta de contrastación (Colom Marañón, 1994). No obstante, ha permitido una primera aproximación de las coordenadas en las que es posible ubicar a la VT a partir de considerar la unión de dos de sus rasgos primarios. 


\section{El Modelo de Personalidad de Cattell.}

Las investigaciones sobre la personalidad de Cattell y sus colegas (Cattell, Eber \& Tatsouka, 1970) aislaron una serie de dimensiones, rasgos o factores de la personalidad a través de tres fuentes de datos: cuestionarios, observaciones en situaciones naturales y tests situacionales objetivos. El cuestionario Sixteen Personality Factor Questionaire (16PF) se diseñó a partir de un análisis lexicográfico basado en una selección de la lista de términos confeccionada por Allport y Odbert (1936). La hipótesis léxica supone que los términos acuñados por el lenguaje natural se refieren a las características de las personas y, en consecuencia, resultan útiles para establecer una taxonomía de la personalidad. Cattell redujo semánticamente la lista obteniendo 35 escalas. Si bien en muestras normales hallaron por análisis factorial 16 rasgos fundamentales primarios, sólo la mitad resultó fácilmente replicable mediante las otras fuentes de datos: A (abierto), B (inteligencia), C (fuerza del ego), E (dominancia), F (seriedad), G (fuerza del super-ego), H (inhibición), I (sensibilidad).

Según los estudios de Cattell el rasgo Fuerza del Super-ego es el mejor predictor del rendimiento académico. Una puntuación baja en esta dimensión es propia de una persona despreocupada, negligente, autocondescendiente y con poca socialización en normas y obligaciones. En cambio, un alto puntaje se asocia a una personalidad escrupulosa, persistente, consciente, disciplinada, metódica, dominada por el sentimiento del deber, moralista y formal. Estos sujetos asumen pronto las responsabilidades sociales y suelen ser estudiantes concienzudos (Colom Marañón, 1995).

Es conveniente destacar la apelación que realizó Cattell a la teoría psicoanalítica para dar nombre a este factor dado que lo suponía como determinante de un poderoso autocontrol y de la persistencia para alcanzar metas ultra-personales e ideales. El nivel en que un sujeto presentaba estas características correspondía a la capacidad del yo para responder a las demandas de un super-yo con mayor o menor grado de severidad. No obstante, Cattell (1972) aseguraba que la Fuerza del Superego no era un determinante exclusivo de la perseverancia sino que este comportamiento también podía resultar de un "intento por satisfacer la ambición personal" (p. 79). Sin embargo, no echó luz acerca del porqué de esta distinción teórica sobre todo teniendo en cuenta que ningún otro factor del $16 \mathrm{PF}$ rescataba este segundo determinante.

Los cuestionamientos más fuertes a este modelo llegaron, como se verá más adelante, de parte de los autores que defendían el modelo pentafactorial. Peabody (1987) advirtió que, al analizar el listado de los términos usado por Cattell, era reconocible una drástica infrarrepresentación de rasgos relacionados con la escrupulosidad y el control de impulsos mientras que estaban sobrerrepresentados rasgos de neuroticismo y cultura. Esto explicaría por qué sólo uno de los 16 factores, y de manera tangencial, da cuenta de aspectos tales como la tenacidad y la responsabilidad. En general, la inmensa mayoría de los trabajos realizados sobre el $16 \mathrm{PF}$ no permitieron confirmar los factores primarios de Cattell (Colom Marañón, 1994).

\section{El Modelo de Personalidad de Eysenck.}

Eysenck y Eysenck (1985) indicaron que la validez de constructo de los instrumentos psicométricos para la medición de la personalidad requería de una teoría que permitiera realizar predicciones complejas en tres áreas: fisiología, experimentos psicológicos y comportamiento social. Utilizando los conceptos neurofisiológicos de reactivación cortical y actividad del cerebro visceral, Eysenck formuló una teoría causal sobre las dimensiones básicas de la personalidad. Su esfuerzo de síntesis sobre las teorías factoriales de las diferencias individuales en personalidad concluyó en el sistema PEN (los superfactores de la personalidad: Psicoticismo, Extraversión y Neuroticismo) que explicaba la mayor parte de la varianza total atribuida al temperamento.

Un análisis de las definiciones dadas por Eysenck permitió suponer que la VT estaría vinculada, aunque parcialmente, con el polo negativo del Psicoticismo. Este factor reúne indicadores asociados a problemas de socialización en donde la impulsividad y el desprecio por las normas ocupan un lugar importante. En lo que respecta a su similitud con VT, un sujeto con bajos niveles de Psicoticismo es considerado como prudente y reflexivo. Pero este factor también permite evaluar la capacidad de 
empatía, la tendencia al respeto y cordialidad en las relaciones interpersonales y el grado de sensibilidad frente a problemas humanitarios.

No obstante, de los tres factores que componen el PEN, la escala de Psicoticismo es la que más problemas ha presentado de confiabilidad y validez (Barrio, 1992). Pelechano y Servando Díaz (2004) reconocieron un intento serio en la teorización de este constructo, pero le atribuyeron una operacionalización pobre, con resultados inconclusos y en su mayoría fuera de la línea prevista por el modelo. Eysenck (1992) justificó los resultados adversos argumentando que la escala Psicoticismo se compone de rasgos relativamente poco relacionados entre sí pero que, en definitiva, sus marcadores comportamentales apuntan a reflejar la fuerza de la conexión entre el individuo y la sociedad.

Si bien son importantes los superfactores como marco general de referencia, a los fines de este análisis era preciso tratar de aislar los factores primarios más próximos al comportamiento de las personas en situación de realizar una tarea.

\section{El Modelo de Personalidad de los Cinco Grandes.}

La teoría pentafactorial es el modelo que actualmente parece dominar el panorama de la evaluación de la personalidad. Una importante ventaja que la destaca de los anteriores modelos es que los resultados no son exclusividad de un autor sino que son dimensiones que han aparecido de modo sistemático en una gran cantidad de estudios.

Dos líneas de investigación con más de 50 años de desarrollos (McCrae \& John, 1992; Sanz, Silva \& Avia, 1999) convergen en señalar que la personalidad puede ser descripta a partir de Cinco Grandes Factores. La más antigua remite al enfoque lexicográfico, que condensa una serie de investigaciones que hallan su origen en la lista de términos de Allport y Odbert (1936). Las primeras divergencias han surgido por la elección del método empleado para reducir este listado. Muchos autores (e.g. Norman, 1963; Tupes \& Christal, 1961) han aprovechado la selección de términos de Cattell (mencionada anteriormente) y consiguieron los cinco dominios del modelo al factorizar las 35 variables que componen el 16PF. Pero en 1967, Norman realizó una nueva reducción y clasificación de los términos al sospechar que las limitaciones técnicas e informáticas de la época podrían haber sesgado la selección de Cattell. Su investigación condujo a una similar solución pentafactorial obtenida tanto con autoinformes como con heterocalificaciones de escalas bipolares.

La segunda línea de investigación encuentra a McCrae y Costa como sus máximos referentes. Estos autores sostienen la existencia de Cinco factores para describir la personalidad sobre la base de estudios factoriales de diversos cuestionarios. El Inventario NEO-PI-R (Costa \& McCrae, 1999), resultado de sus elaboraciones, los ha llevado a concluir recientemente la universalidad de los cinco factores tras estudiar la replicación de la estructura en 50 culturas (McCrae \& Terracciano, 2005).

Uno de los cinco dominios que comprenden este modelo incluye, junto con otros aspectos, las características primordiales de la VT. Más allá de algunas divergencias teóricas, los autores alineados en este modelo han llegado a definir con el vocablo Escrupulosidad (Conscientiousness) al grado en que un sujeto puede autorregularse. Con este término se refieren tanto a su posibilidad de controlar los impulsos así como también su capacidad para generar conductas proactivas tendientes a la planificación, organización y resolución de tareas (Costa \& McCrae, 1999). De igual forma se han empleado otras denominaciones como Tesón (Caprara, Barbaranelli \& Borgogni, 1993), Trabajo (Work) (Peabody \& Goldberg, 1989) o incluso la notación romana III surgida de las investigaciones de Norman (1967). Todas estas alusiones responden a constructos similares a los que se ha arribado en diferentes operacionalizaciones. Los nombres asignados a este constructo se multiplican al revisar las traducciones que presenta la literatura en castellano: Consciencia (Barrio, 1992), Minuciosidad (Colom Marañón, 1995), Escrupulosidad (Pelechano, 1996) y Responsabilidad (Avia 1995).

Sin embargo, Sanz et al. (1999) advirtieron que las diferentes denominaciones a veces encubrían divergencias conceptuales que no debían soslayarse. Al comparar el parecido de las definiciones conceptuales en las diferentes investigaciones se encontró que, si bien estaban emparentadas, no 
eran idénticas. A iguales conclusiones han llegado Pelechano y Servando Díaz (2004); quienes aseguraron que, aunque los autores coincidieran en referir al dominio como Escrupulosidad, las facetas psicológicas que abarcaba cada definición eran muy diferentes. Barrick y Mount (1991) señalaron que la principal distinción que se podía apreciar entre los autores respondía a un desacuerdo que concernía a la esencia misma de esta dimensión. Algunos consideraban que la Escrupulosidad respondía únicamente a la capacidad de autocontrol de los impulsos de un sujeto. Bajo esta definición surgieron denominaciones tales como Prudencia (Prudence) (Hogan, 1986) o Formalidad (Dependability) (Tupes \& Christal, 1961) que enfatizaban la mirada sobre la tendencia del individuo a ser cuidadoso y perfeccionista con las tareas que realiza así como también su capacidad para organizar y planificar las actividades. En cambio, otros autores suponían que la escrupulosidad debía ser entendida no solo como la inhibición de un comportamiento impulsivo, sino que comprendía además la autorregulación de las conductas proactivas tendientes a la concreción de las tareas (Costa \& McCrae, 1999; Digman, 1990). Es por esta razón que incorporaron a este constructo características que describían la perseverancia, la búsqueda activa de logros y la posibilidad de trabajar duramente. En virtud de su evidente relación con la búsqueda y obtención de logros laborales y académicos comenzaron a surgir denominaciones tales como Voluntad de Logros (Will to Achieve) (Digman \& Takemoto-Chock, 1981) o Tesón (Caprara et al. 1993).

Una de las críticas más fuertes acerca de la unidad de Escrupulosidad llegó de parte de Paunonen y Jackson (1996). Según sus investigaciones, aplicando el Jackson Personality Inventory (Jackson, 1984) los elementos que componían este dominio no podían agruparse en un único factor. Para ellos el constructo estaba integrado por tres dimensiones que debían interpretarse de manera aislada aunque presentaran cierto solapamiento: a) meticulosidad y orden, b) formalidad y fiabilidad, y c) ambición y búsqueda de éxito. En la misma línea, Hogan y Hogan (1992) ubicaban la Prudencia y Ambición (Ambition) como dos factores independientes. Por su parte, Costa y McCrae (1998) sostenían que estas críticas no repercutían en su modelo porque estos autores partían de una estructuración de la personalidad ligeramente diferente que hacía que sus conceptualizaciones no fueran equiparables.

A los fines de esta delimitación, tanto las divergencias internas al modelo como los cuestionamientos que lo atacaron desde fuera resultaron cruciales. En efecto, ambos permitieron un análisis más sutil de los componentes de Escrupulosidad. Dicho análisis sirvió para aislar el conjunto de aspectos que, pese a ser representativos del constructo, pudieron entenderse como subdimensiones solidarias a las conductas que englobaban la idea de VT. Indudablemente, VT se vinculaba exclusivamente con el aspecto proactivo de la autorregulación, lo cual deja por fuera los indicadores asociados a la capacidad de planificación y orden. A su vez, de la proactividad se descartaron la propensión a la búsqueda constante de logros y la ambición ya que no eran características consistentes con la definición de VT. De esta manera, la VT ha quedado circunscripta a los atributos que diferencian a los sujetos en su capacidad de trabajar duramente y en su constancia para empezar, continuar y concluir una tarea.

\section{El Modelo de Personalidad de los Cinco Alternativos de Zuckerman.}

El planteo de Zuckerman abrió juicio sobre el enfoque lexicográfico al cuestionar que los términos surgidos del lenguaje natural no necesariamente tenían que reflejar la importancia que una dimensión básica presentaba en la estructura de la personalidad. Su modelo factorial fue el resultado del análisis de diversas escalas que medían constructos con comprobado basamento psicobiológico. Como consecuencia de estas investigaciones Zuckerman ha construido el cuestionario ZKPQ, compuesto por 50 ítems que han sido adaptados y validados en estudios transculturales (Aluja et al., 2006). El modelo reconoce cinco dimensiones básicas de la personalidad: Neuroticismo-Ansiedad, Búsqueda Impulsiva de Sensaciones, Agresión-Hostilidad, Actividad y Sociabilidad.

El aporte que este modelo realizó al presente estudio permitió repensar el mismo constructo siendo observado desde otra perspectiva. Para el modelo de Zuckerman, la VT se encontraría vinculada desde un plano teórico con el factor denominado Actividad. Este componente describe la incapacidad para permanecer inactivo y la necesidad de buscar tareas que permitan utilizar la energía. 
Contiene dos subescalas: una que recoge aspectos más generales de la actividad y otra que es más específica llamada Esfuerzo de Trabajo (Work Effort) y se refiere a la preferencia por los desafíos y el trabajo duro así como también a la cantidad de energía que es aplicada al trabajo y otras tareas.

Como es posible apreciar, en el abordaje de Zuckerman se ha hecho más hincapié en lo que desde el Modelo de los Cinco Factores se denomina proactividad. La mayoría de los modelos de personalidad se han centrado en los procesos reguladores de la conducta mientras que Zuckerman ha puesto el acento en la conducta y en la energía disponible para llevarla adelante. De la energía total con la que cuenta el sujeto para sus actividades, se aísla una parte que puede ser encaminada para cumplir con los objetivos de su trabajo. De esta manera ha permitido vislumbrar la contracara del mismo constructo.

Sin embargo, cabe aclarar que la subescala Esfuerzo de Trabajo ha evidenciado ciertas limitaciones psicométricas. Si bien Zuckerman (2002) informó un alfa de Cronbach de 0.75 para hombres y 0.62 para mujeres, también aseguró que la delimitación de las subescalas era aún provisoria en su modelo y que requería de sucesivas investigaciones que mostraran la invariancia de esta estructura. En consonancia con esta aclaración, algunos estudios españoles independientes han registrado alfas de Cronbach particularmente bajos para esta subescala de 0.47 para población adulta (Zotes, 1999) y 0.40 para adolescentes (Romero, Luengo, Gómez-Fraguela \& Sobral, 2002).

\section{Discusión}

La Voluntad ha tenido múltiples formulaciones a lo largo de su historia. En esta delimitación de la Voluntad de Trabajo se han unido los aportes de las corrientes filosóficas y psicológicas. Se partió desde la Filosofía con la idea de Voluntad como motor para iniciar, continuar o terminar las acciones. Se han reseñado los primeros intentos de experimentación con el objetivo de mostrar la evolución de los fundamentos teóricos de los actuales Modelos de Control de la Acción. Estos alcanzaron una definición específica de Voluntad al insertarla en un modelo motivacional más amplio. Así, diferenciaron entre la intencionalidad de la conducta (las tendencias motivacionales que llevan al sujeto a perseguir la meta que se ha propuesto) y la mediación de los procesos volitivos para hacerla operativa. Estos aportes han permitido entender a la Voluntad como el proceso psíquico que permite encauzar los impulsos que componen la intención limitando la influencia de tendencias contrarias, es decir, como el conjunto de mecanismos que regulan la conducta para lograr la superación de los obstáculos y el mantenimiento de la intención desde el planteo del objetivo hasta su concreción. Se ha considerado, siguiendo la clasificación de Rojas (2003), que pueden identificarse una voluntad inicial que pone en marcha al individuo, una voluntad perseverante que sostiene la intención a lo largo de su desarrollo y una voluntad ejemplar que permite concluir el trabajo atendiendo a cada uno de los detalles. En virtud de los objetivos de la presente investigación, dentro de todas las áreas a las que puede referirse la Voluntad, se ha restringido el alcance del constructo a toda actividad productiva, sea o no retribuida.

El estudio de los Modelos de Personalidad ha permitido reconocer las dimensiones que se refieren a la VT en la literatura psicométrica y los indicadores conductuales más frecuentemente utilizados para describir esta variable. Se observó que con distintos nombres y correspondiendo a escalas o subescalas más o menos equivalentes entre sí, son muchos los modelos de personalidad que han logrado identificar componentes que dan cuenta de la perseverancia y disposición al esfuerzo de un sujeto para cumplir con sus deberes. Ahora bien, existen matices diferenciales que sólo es posible apreciar al establecer comparaciones entre los distintos enfoques. Autores como Cattell o Eysenck hacían hincapié en la relación del sujeto con otros individuos. Desde estas perspectivas, la responsabilidad era vista como la capacidad del sujeto para responder en tiempo y forma con sus obligaciones evitando el perjuicio que pudiera ocasionar a los otros si no las cumpliera. Es decir, estos inventarios evalúan las conductas socialmente esperables para mantener las normas de convivencia. Otros autores, entre los que se encuentran Guilford, Zuckerman y los investigadores que proponen los Cinco Grandes, fueron más restrictivos al buscar objetivar concretamente la relación del sujeto con la tarea que realiza. De esta manera, generaron indicadores mucho más próximos a las conductas concretas de las personas sin plantear las repercusiones que éstas poseían en el campo social. Cada opera- 
cionalización refleja una definición diferente de VT que infra o supra representa distintos aspectos de la misma. Por ejemplo, en los inventarios correspondientes a los Cinco Grandes, la dimensión Escrupulosidad presenta múltiples facetas pero muy pocos indicadores reflejan la VT (trabajo duro y perseverancia). Otras subdimensiones que se refieren a las capacidades de planificación, orden, atención, tolerancia a la frustración, búsqueda de logro, decisión, prudencia, adhesión a principios éticos exceden lo que se pretende estudiar aquí. En el cuestionario GZTS no hay una escala concreta que evalúe la VT sino que este constructo se encuentra confundido entre los rasgos primarios de Actividad General y Retraimiento. Por otro lado, la escala Esfuerzo de Trabajo de Zuckerman, si bien presenta indicadores de lo que la Teoría del Control de la Acción denomina Voluntad, estos son muy pocos y se encuentran mezclados con otros que se refieren a la búsqueda de logros.

En conclusión, la integración de los aportes hasta aquí desarrollados permiten concebir la VT como la tendencia por la cual un sujeto se aboca a sus obligaciones llevándolas a cabo con responsabilidad, motivado por su propio deseo de realizar un buen trabajo sin rehuir el esfuerzo que ello implica, aun en los casos en que dichas obligaciones pudieran no ser de su agrado. Esto se explica por su capacidad para generar procesos volitivos que le permitan comprometerse activamente en el inicio de un trabajo, persistir en su concreción sin que las tendencias contrarias logren desviarlo de su objetivo y concluirla con precisión y sin dilaciones.

Esta definición conceptual del constructo alcanzada permitirá determinar el tipo de ítems a construir para elaborar una escala que posibilite su evaluación. Los mismos deberán reflejar el repertorio de conductas relevantes a la definición de VT y diferenciar a los sujetos con distinto nivel en el rasgo. Esto garantizará la validez de constructo. Los trabajos futuros estarán encaminados a desarrollar una prueba corta y unidimensional para evaluar este rasgo en la población de alumnos universitarios. Más adelante se intentará su aplicación a la población general.

\section{Referencias}

Abbagnano, N. (1974). Diccionario de Filosofía. México: Fondo de Cultura Económica.

Ach, N. (1910). Über den Willensakt und das Temperament. Liepzig: Quelle und Meyer.

Allport, G. \& Odbert, H. (1936). Trait names: A psycho-lexical study. Psychological Monographs, 47, 171.

Alonso-Tapia, J. (1992). Evaluación de la motivación. En R. Fernández-Ballesteros (Ed.), Introducción a la evaluación psicológica (Vol. 2, pp. 157-203). Madrid: Pirámide.

Aluja, A., Rossier, J. García, L., Angleitner, A, Kuhlman, M. \& Zuckerman, M. (2006). A crosscultural shortened form of the ZKPQ (ZKPQ-50-cc) adapted to English, French, German, and Spanish languages. Personality and Individual Differences, 41, 619-628.

Arana, J. M. \& Mateos, P. M. (1997). Intencionalidad y memoria: el mantenimiento y protección de las intenciones pospuestas ante la aparición de obstáculos a la acción. Estudios de Psicología, $58,29-42$.

Arana, J. M. \& Sanfeliu, M. C. (1994). Avatares históricos del concepto de volición desde el inicio de su estudio en la psicología experimental hasta nuestros días. Revista de Historia de la Psicología, 15(3-4), 427-438.

Avia, M. D. (1995). Los rasgos de personalidad: Estructuras empíricas y representaciones cognitivas. En M. D. Avia \& M. L. Sánchez-Bernardos (Eds.), Personalidad: Aspectos cognitivos y sociales (pp.287-294). Madrid: Pirámide.

Barberá, E. (1995). Establecimiento de metas en la conducta motivada: perspectiva histórica. Revista de Historia de la Psicología, 3-4(16), 103-110.

Barberá, E. (2000). Marco conceptual e investigación de la motivación humana. Revista Española de Motivación y Emoción, 1, 23-36. 
Barrick, M. R. \& Mount, M. K. (1991). The Big Five personality dimensions and job performance: a meta-analysis. Personnel Psychology, 44, 1-26.

Barrio, V del (1992). Evaluación de características de la personalidad. En R. Fernández-Ballesteros (Ed.), Introducción a la evaluación psicológica (Vol. 2, pp. 17-64). Madrid: Pirámide.

Caprara, G.V., Barbaranelli, C. \& Borgogni, L. (1993). Big Five Questionnaire (BFQ). Manuale. Florencia: Organizzazioni Speciali.

Cattell, R. B. (1972). El análisis científico de la personalidad. Barcelona: Fontanella.

Cattell, R. B., Eber, H. W. \& Tatsouka, M. M. (1970). Handbook for the Sixteen Personality Factor Questionnaire (16PF). Institute for personality and ability testing, Champaign III.

Colom Marañón, R. (1994). Psicología de las Diferencias Individuales. Madrid: Pirámide.

Colom Marañón, R. (1995). Tests, Inteligencia y personalidad. Madrid: Pirámide.

Costa, P. T., Jr. \& McCrae, R. R. (1998). Six approaches to the explication of facet-level traits: examples from conscientiousness. European Journal of Personality, 12, 117-134.

Costa, P.T. \& McCrae, R. R. (1999). Manual NEO PI-R, Inventario de Personalidad NEO Revisado y NEO-FFI, Inventario NEO reducido de Cinco Factores. Madrid: TEA Ediciones, S.A.

Digman, J. M. (1990). Personality structure: Emergence of the five-factor model. Annual review of psychology, 51, 417-440.

Digman, J. M. \& Takemoto-Chock, N. K. (1981). Factors in the natural language of personality: Reanalysis, comparison and interpretation of six major studies. Multivariate Behavoiral Research, $16,149-170$.

Dorsch, F. (1991). Diccionario de Psicología. Barcelona: Editorial Herder.

Eysenck, H. J. (1992). The definition and measurement of psychoticism. Personality and Individual Differences, 13, 757-785.

Eysenck, H. J. \& Eysenck, M. (1985): Personalidad y Diferencias Individuales. Madrid: Pirámide.

Ferrater Mora, J. (1975). Diccionario de Filosofía. Buenos Aires: Sudamericana.

Guilford, J. P. \& Zimmerman, W. S. (1949). Guilford-Zimmermann Temperament Survey: Manual. Beverly Hills, California: Sheridan Supply.

Guilford, J. P. (1959). Personality. Nueva York: McGraw-Hill.

Guilford, J. P. (1975). Factors and factors of personality, Psychological Bulletin, 82(5), 802-814.

Heckhausen, H. (1987). Jenseits des Rubikon: Der Wille inden Human Wissenschaften. Berlín: Springer-Verlag.

Hogan, R. (1986). Hogan Personality Inventory Manual. Minneapolis, MN: National Computer Systems.

Hogan, R. \& Hogan, J. (1992). Hogan Personality Inventory Manual. Tulsa, OK: Hogan Assessment Systems.

Jackson, D. N. (1984). Personality Research Form Manual (3 ${ }^{\mathrm{a}}$ ed.). Port Huron, MI: Research Psychologists Press.

Karoly, P. (1993). Mechanisms of self-regulation: A systems view. Annual Review of Psychology, 44, 23-52.

Kuhl, J. (1985). Volitional mediators of cognition behavior consistency: Self-regulatory processes and action versus state orientation. En J. Kuhl y J. Beckman (Eds.), Action control: From cognition to behavior. Nueva York: Springer-Verlag. 
McClelland, D. C. (1961). The achieving society. Princeton, NJ: Van Nostrand.

McCrae, R. R. \& John, O. P. (1992). An introduction to the five-factor model and its applications. Journal of Personality, 60, 175-215.

McCrae, R. R. \& Terracciano, A. (2005). Universal Features of Personality Traits From the Observer's Perspective: Data From 50 Cultures. Journal of Personality and Social Psychology, 88(3), 547-561.

Michotte, A. \& Prüm, E. (1910). Le Choix Volontaire et ses antécédents immédiats, Arch de Psychol, 38-39, 8-205.

Norman W. T. (1963). Toward an adequate taxonomy of personality attibutes: Replicated factor structure in peer nomination personality ratings. Journal of Abnormal and Social Psychology, 66, 574-583.

Norman W. T. (1967). 2800 personality trait descriptors: Normative operating characteristics for university population. Ann Arbor: Department of Psychology, University of Michigan.

Paunonen, S. y Jackson, D. N. (1996). The Jackson Personality Inventory and the Five-Factor Model of personality. Journal of Research in Personality, 30, 42-59.

Peabody, D. (1987) Selecting representative trait adjectives. Journal of Personality and Social Psychology, 52, 59-71.

Peabody, D. \& Goldberg, L.R. (1989). Some determinants of factor structures from personality-trait descriptors. Journal of Personality and Social Psychology, 57, 552-567.

Peiró, J.; Prieto, F. \& Roe, R. (1996). El trabajo como fenómeno psicosocial. En J. Peiró \& F. Prieto (Eds), Tratado de Psicología del Trabajo Volumen II: Aspectos Psicosociales del Trabajo. Madrid: Editorial Síntesis.

Pelechano, V. (1996). El análisis lingüístico contemporáneo: Los "grandes". En V. Pelechano (Ed.), Psicología de la Personalidad I: Teorías (pp. 89-109) Barcelona: Ariel.

Pelechano, V. \& Servando Díaz, M. A. (2004). Qué es la personalidad. Madrid: Biblioteca Nueva.

Rojas, E. (2003). La conquista de la voluntad. Buenos Aires: Editorial Planeta.

Romero, E., Luengo, M. A., Gómez-Fraguela, J. A. \& Sobral, J. (2002). La estructura de la personalidad en adolescentes: El Modelo de Cinco Factores y los Cinco Alternativos. Psicothema, 14(1), 134-143.

Sanz, J.; Silva, F. \& Avia, M. D. (1999). La evaluación de la personalidad desde el modelo de los "Cinco Grandes": El Inventario de Cinco-Factores NEO de Costa y McCrae. En F. Silva (Ed.), Avances en Evaluación Psicológica. Valencia: Promolibro.

Tupes, E. C. \& Christal, R. E. (1961). Recurrent personality factors based on trait ratings (Tech. Rep No 61-97). Lackland Air Fource Base, TX: Aeronautical Systems Division.

Zotes, J.A. (1999). Cuestionario de Personalidad Zuckerman-Kuhlman (ZKPQIII; 1993). Manuscrito no publicado. Hospital Ramón y Cajal, Madrid.

Zuckerman, M. (2002). Zuckerman-Kuhlman personality questionnaire (ZKPQ): an alternative five-factorial model. En B. De Raad \& M. Perugini (Eds.), Big five assessment (pp. 377-396). Seattle: Hogrefe and Huber Publishers. 